\title{
Search for excited leptons in $\mathrm{e}^{+} \mathrm{e}^{-}$interactions at $\sqrt{s}=192-202 \mathrm{GeV}$
}

\section{L3 Collaboration}

M. Acciarri ${ }^{\mathrm{y}}$, P. Achard ${ }^{\mathrm{s}}$, O. Adriani ${ }^{\mathrm{p}}$, M. Aguilar-Benitez ${ }^{\mathrm{x}}$, J. Alcaraz $^{\mathrm{x}}$, G. Alemanni ${ }^{\text {u }}$, J. Allaby ${ }^{\mathrm{q}}$, A. Aloisio ${ }^{\text {aa }}$, M.G. Alviggi ${ }^{\text {aa }}$, G. Ambrosi ${ }^{\text {s }}$, H. Anderhub ${ }^{\text {av }}$, V.P. Andreev f,ai , T. Angelescu ${ }^{1}$, F. Anselmo ${ }^{\mathrm{i}}$, A. Arefiev ${ }^{\mathrm{z}}$, T. Azemoon ${ }^{\mathrm{c}}$, T. Aziz ${ }^{\mathrm{j}}$, P. Bagnaia ah , A. Bajo ${ }^{\text {x }}$, L. Baksay ${ }^{\text {aq }}$, A. Balandras ${ }^{d}$, S.V. Baldew ${ }^{b}$, S. Banerjee ${ }^{j}$, Sw. Banerjee ${ }^{j}$, A. Barczyk ${ }^{\text {av, at }}$, R. Barillère ${ }^{q}$, P. Bartalini ${ }^{u}$, M. Basile ${ }^{i}$, N. Batalova ${ }^{\text {as }}$, R. Battiston ${ }^{\text {ae }}$, A. Bay ${ }^{\mathrm{u}}$, F. Becattini ${ }^{\mathrm{p}}$, U. Becker ${ }^{\mathrm{n}}$, F. Behner ${ }^{\text {av }}$, L. Bellucci $^{\mathrm{p}}$, R. Berbeco ${ }^{\text {c }}$, J. Berdugo ${ }^{\mathrm{x}}$, P. Berges ${ }^{\mathrm{n}}$, B. Bertucci ${ }^{\text {ae }}$, B.L. Betev ${ }^{\text {av }}$, S. Bhattacharya ${ }^{\mathrm{j}}$, M. Biasini ${ }^{\text {ae }}$, A. Biland ${ }^{\text {av }}$, J.J. Blaising ${ }^{\text {d }}$, S.C. Blyth ${ }^{\text {af }}$, G.J. Bobbink ${ }^{\text {b }}$, A. Böhm ${ }^{\text {a }}$, L. Boldizsar ${ }^{\mathrm{m}}$, B. Borgia ${ }^{\text {ah }}$, D. Bourilkov ${ }^{\text {av }}$, M. Bourquin ${ }^{\mathrm{s}}, \mathrm{S}$. Braccini ${ }^{\mathrm{s}}$, J.G. Branson $^{\text {am }}$, F. Brochu ${ }^{\text {d }}$, A. Buffini ${ }^{\mathrm{p}}$, A. Buijs ${ }^{\text {ar }}$, J.D. Burger ${ }^{\mathrm{n}}$, W.J. Burger ${ }^{\text {ae }}$, X.D. Cai ${ }^{\text {n}}$, M. Capell ${ }^{\text {n}}$, G. Cara Romeo ${ }^{\mathrm{i}}$, G. Carlino ${ }^{\text {aa }}$, A.M. Cartacci ${ }^{\mathrm{p}}$, J. Casaus ${ }^{\mathrm{x}}$, G. Castellini $^{\text {p }}$, F. Cavallari ${ }^{\text {ah }}$, N. Cavallo ${ }^{\text {aj }}$, C. Cecchi ${ }^{\text {ae }}$, M. Cerrada ${ }^{\mathrm{x}}$, F. Cesaroni ${ }^{\mathrm{v}}$, M. Chamizo ${ }^{\text {s, Y.H. Chang }}{ }^{\text {ax }}$, U.K. Chaturvedi ${ }^{r}$, M. Chemarin ${ }^{w}$, A. Chen ${ }^{\text {ax }}$, G. Chen ${ }^{\mathrm{g}}$, G.M. Chen ${ }^{\mathrm{g}}$, H.F. Chen ${ }^{\mathrm{t}}$, H.S. Chen ${ }^{\mathrm{g}}$, G. Chiefari ${ }^{\text {aa }}$, L. Cifarelli ${ }^{\text {al }}$, F. Cindolo ${ }^{\mathrm{i}}$, C. Civinini ${ }^{\mathrm{p}}, \mathrm{I}_{\text {. Clare }}{ }^{\mathrm{n}}$, R. Clare ${ }^{\mathrm{ak}}, \mathrm{G}$ Coignet $^{\mathrm{d}}$, N. Colino $^{\mathrm{x}}$, S. Costantini ${ }^{\mathrm{e}}$, F. Cotorobai ${ }^{1}$, B. de la Cruz $^{\mathrm{x}}$, A. Csilling ${ }^{\mathrm{m}}$, S. Cucciarelli ${ }^{\text {ae }}$, T.S. Dai ${ }^{\mathrm{n}}$, J.A. van Dalen ${ }^{\text {ac }}$, R. D’Alessandro ${ }^{\mathrm{p}}$, R. de Asmundis ${ }^{\text {aa }}$, P. Déglon ${ }^{\mathrm{s}}$, A. Degré ${ }^{\mathrm{d}}$, K. Deiters ${ }^{\text {at }}$, D. della Volpe ${ }^{\text {aa }}$, E. Delmeire ${ }^{\text {s }}$, P. Denes ${ }^{\text {ag }}$, F. DeNotaristefani ${ }^{\text {ah }}$, A. De Salvo ${ }^{\text {av }}$, M. Diemoz ${ }^{\text {ah }}$, M. Dierckxsens ${ }^{\text {b }}$, D. van Dierendonck ${ }^{\text {b }}$, C. Dionisi ${ }^{\text {ah }}$, M. Dittmar av , A. Dominguez ${ }^{\text {am }}$, A. Doria ${ }^{\text {aa }}$, M.T. Dova ${ }^{\text {r,5 }}$, D. Duchesneau ${ }^{\text {d, }}$ D. Dufournaud ${ }^{\text {d }}$, P. Duinker ${ }^{\text {b }}$, I. Duran ${ }^{\text {an }}$, H. El Mamouni ${ }^{\text {w }}$, A. Engler ${ }^{\text {af }}$, F.J. Eppling ${ }^{\text {n }}$, F.C. Erné ${ }^{\mathrm{b}}$, A. Ewers ${ }^{\mathrm{a}}$, P. Extermann ${ }^{\mathrm{s}}$, M. Fabre ${ }^{\text {at }}$, M.A. Falagan ${ }^{\mathrm{x}}$, S. Falciano ${ }^{\text {ah, }}{ }^{\text {, }}$, A. Favara ${ }^{\text {q }}$, J. Fay ${ }^{\text {w }}$, O. Fedin ${ }^{\text {ai }}$, M. Felcini ${ }^{\text {av }}$, T. Ferguson ${ }^{\text {af }}$, H. Fesefeldt ${ }^{\text {a }}$, E. Fiandrini ${ }^{\text {ae }}$, J.H. Field ${ }^{\text {s }}$, F. Filthaut ${ }^{\mathrm{q}}$, P.H. Fisher ${ }^{\mathrm{n}}$, I. Fisk ${ }^{\text {am }}$, G. Forconi ${ }^{\text {n, }}$, K. Freudenreich ${ }^{\text {av }}$, C. Furetta ${ }^{y}$, Yu. Galaktionov ${ }^{z, n}$, S.N. Ganguli ${ }^{j}$, P. Garcia-Abia ${ }^{\mathrm{e}}$, M. Gataullin ${ }^{\text {ad }}$, S.S. Gau ${ }^{\text {k }}$, S. Gentile ${ }^{\text {ah, }}$, N. Gheordanescu ${ }^{1}$, S. Giagu ${ }^{\text {ah }}$, Z.F. Gong ${ }^{\text {, }}$, G. Grenier ${ }^{\text {w }}$, O. Grimm ${ }^{\text {av }}$, M.W. Gruenewald ${ }^{\mathrm{h}}$, M. Guida ${ }^{\text {al }}$, R. van Gulik ${ }^{\mathrm{b}}$, V.K. Gupta ${ }^{\text {ag }}$, A. Gurtu ${ }^{\text {j, L.J. Gutay }}{ }^{\text {as }}$, D. Haas ${ }^{\mathrm{e}}$, A. Hasan ${ }^{\mathrm{ab}}$, D. Hatzifotiadou ${ }^{\mathrm{i}}$, T. Hebbeker ${ }^{\text {h}}$, A. Hervé ${ }^{\mathrm{q}}$, P. Hidas ${ }^{\mathrm{m}}$, J. Hirschfelder ${ }^{\text {af }}$, H. Hofer ${ }^{\text {av }}$, G. Holzner ${ }^{\text {av }}$, 
H. Hoorani ${ }^{\text {af }}$, S.R. Hou ${ }^{\text {ax }}$, Y. Hu ${ }^{\text {ac }}$, I. Iashvili ${ }^{\text {au }}$, B.N. Jin ${ }^{\mathrm{g}}$, L.W. Jones ${ }^{\mathrm{c}}$, P. de Jong ${ }^{\mathrm{b}}$, I. Josa-Mutuberría ${ }^{\mathrm{x}}$, R.A. Khan ${ }^{\mathrm{r}}$, D. Käfer ${ }^{\mathrm{a}}$, M. Kaur ${ }^{\mathrm{r}, 6}$, M.N. Kienzle-Focacci ${ }^{\mathrm{s}}$,

D. Kim ${ }^{\text {ah }}$, J.K. Kim ${ }^{\text {ap }}$, J. Kirkby ${ }^{\text {q }}$, D. Kiss ${ }^{\mathrm{m}}$, W. Kittel ${ }^{\text {ac }}$, A. Klimentov ${ }^{\text {n,z }}$, A.C. König ac ${ }^{\text {ac }}$ M. Kopal ${ }^{\text {as }}$, A. Kopp ${ }^{\text {au }}$, V. Koutsenko ${ }^{\text {n,z }}$, M. Kräber ${ }^{\text {av }}$, R.W. Kraemer ${ }^{\text {af }}$, W. Krenz ${ }^{\mathrm{a}}$, A. Krüger ${ }^{\text {au }}$, A. Kunin ${ }^{\mathrm{n}, \mathrm{z}}$, P. Ladron de Guevara ${ }^{\mathrm{x}}$, I. Laktineh ${ }^{\mathrm{w}}$, G. Landi ${ }^{\mathrm{p}}$, M. Lebeau ${ }^{\mathrm{q}}$, A. Lebedev ${ }^{\mathrm{n}}$, P. Lebrun ${ }^{\mathrm{w}}$, P. Lecomte ${ }^{\text {av }}$, P. Lecoq ${ }^{\mathrm{q}}$, P. Le Coultre ${ }^{\text {av }}$, H.J. Lee ${ }^{\text {h }}$, J.M. Le Goff ${ }^{\mathrm{q}}$, R. Leiste ${ }^{\text {au }}$, P. Levtchenko ${ }^{\text {ai }}$, C. Li $^{\mathrm{t}}$, S. Likhoded ${ }^{\text {au }}$, C.H. Lin ${ }^{\text {ax }}$, W.T. Lin ${ }^{\text {ax }}$, F.L. Linde ${ }^{\text {b }}$, L. Lista ${ }^{\text {aa }}$, Z.A. Liu ${ }^{\text {g }, ~ W . ~ L o h m a n n ~}{ }^{\text {au }}$, E. Longo ${ }^{\text {ah }}$, Y.S. Lu ${ }^{g}$, K. Lübelsmeyer ${ }^{\text {a }}$, C. Luci ${ }^{\text {q,ah }}$, D. Luckey ${ }^{\mathrm{n}}$, L. Lugnier ${ }^{\mathrm{w}}$, L. Luminari ${ }^{\text {ah }}$, W. Lustermann ${ }^{\text {av }}$, W.G. Ma ${ }^{\mathrm{t}}$, M. Maity ${ }^{\mathrm{j}}$, L. Malgeri ${ }^{\mathrm{q}}$, A. Malinin ${ }^{\mathrm{q}}$, C. Maña ${ }^{\mathrm{x}}$, D. Mangeol ac , J. Mans ${ }^{\text {ag }}$, G. Marian ${ }^{\text {a }}$, J.P. Martin ${ }^{\text {w}}$, F. Marzano ${ }^{\text {ah }}$, K. Mazumdar ${ }^{\mathrm{j}}$, R.R. McNeil ${ }^{\text {f }}$, S. Mele ${ }^{q}$, L. Merola ${ }^{\text {aa }}$, M. Meschini ${ }^{p}$, W.J. Metzger ${ }^{\text {ac }}$, M. von der Mey ${ }^{a}$, A. Mihul ${ }^{1}$, H. Milcent ${ }^{\mathrm{q}}$, G. Mirabelli ah ${ }^{\text {a }}$ J. Mnich ${ }^{\mathrm{a}}$, G.B. Mohanty ${ }^{\mathrm{j}}$, T. Moulik ${ }^{\mathrm{j}}$, G.S. Muanza ${ }^{\text {w }}$, A.J.M. Muijs ${ }^{\mathrm{b}}$, B. Musicar ${ }^{\mathrm{am}}$, M. Musy ${ }^{\text {ah }}$, M. Napolitano ${ }^{\text {aa }}$, F. Nessi-Tedaldi ${ }^{\text {av }}$, H. Newman ${ }^{\text {ad }}$, T. Niessen ${ }^{\text {a }}$, A. Nisati ${ }^{\text {ah }}$, H. Nowak ${ }^{\text {au }}$, R. Ofierzynski ${ }^{\text {av }}$, G. Organtini ${ }^{\text {ah }}$, A. Oulianov ${ }^{z}$, C. Palomares ${ }^{\mathrm{x}}$, D. Pandoulas a ${ }^{\text {a }}$, S. Paoletti ah, ${ }^{\text {a }}$, P. Paolucci ${ }^{\text {aa }}$, R. Paramatti ${ }^{\text {ah }}$, H.K. Park ${ }^{\text {af }}$, I.H. Park ${ }^{\text {ap }}$, G. Passaleva ${ }^{\mathrm{q}}$, S. Patricelli ${ }^{\text {aa }}$, T. Paul ${ }^{\mathrm{k}}$, M. Pauluzzi ${ }^{\text {ae }}$, C. Paus ${ }^{\mathrm{q}}$, F. Pauss ${ }^{\text {av }}$, M. Pedace ${ }^{\text {ah }}$, S. Pensotti ${ }^{y}$, D. Perret-Gallix ${ }^{d}$, B. Petersen ${ }^{\text {ac }}$, D. Piccolo ${ }^{\text {aa }}$, F. Pierella ${ }^{\text {i }}$, M. Pieri ${ }^{\mathrm{p}}$, P.A. Piroué ${ }^{\mathrm{ag}}$, E. Pistolesi ${ }^{\mathrm{y}}$, V. Plyaskin ${ }^{\mathrm{z}}$, M. Pohl ${ }^{\mathrm{s}}$, V. Pojidaev ${ }^{\mathrm{z}, \mathrm{p}}$,

H. Postema ${ }^{\mathrm{n}}$, J. Pothier ${ }^{\mathrm{q}}$, D.O. Prokofiev as ${ }^{\text {, D. Prokofiev }}{ }^{\text {ai }}$, J. Quartieri ${ }^{\text {al }}$, G. Rahal-Callot ${ }^{\mathrm{av}, \mathrm{q}}$, M.A. Rahaman ${ }^{\mathrm{j}}$, P. Raics ${ }^{\mathrm{o}}$, N. Raja ${ }^{\mathrm{j}}$, R. Ramelli ${ }^{\text {av }}$, P.G. Rancoita ${ }^{\mathrm{y}}$, R. Ranieri ${ }^{\mathrm{p}}$, A. Raspereza ${ }^{\mathrm{au}}$, G. Raven ${ }^{\mathrm{am}}$, P. Razis $^{\mathrm{ab}},{ }^{\mathrm{D}}$ D. Ren ${ }^{\mathrm{av}}, \mathrm{M}$. Rescigno ${ }^{\text {ah }}$, S. Reucroft ${ }^{\mathrm{k}}$, S. Riemann ${ }^{\text {au }}, \mathrm{K}$. Riles ${ }^{\mathrm{c}}$, J. Rodin ${ }^{\mathrm{aq}}$, B.P. Roe ${ }^{\mathrm{c}}$, L. Romero ${ }^{\mathrm{x}}$, A. Rosca ${ }^{\mathrm{h}}$, S. Rosier-Lees ${ }^{d}$, S. Roth ${ }^{a}$, C. Rosenbleck ${ }^{a}$, J.A. Rubio ${ }^{q}$, G. Ruggiero ${ }^{p}$, H. Rykaczewski ${ }^{\text {av }}$, S. Saremi ${ }^{\text {f }}$, S. Sarkar ${ }^{\text {ah }}$, J. Salicio ${ }^{\text {q }}$, E. Sanchez ${ }^{\text {q }}$, M.P. Sanders ${ }^{\text {ac }}$,

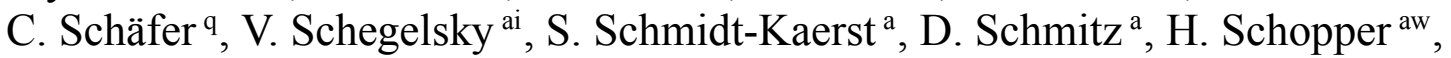
D.J. Schotanus ${ }^{\text {ac }}$, G. Schwering ${ }^{\text {a }}$, C. Sciacca ${ }^{\text {aa }}$, A. Seganti ${ }^{i}$, L. Servoli ${ }^{\text {ae }}$, S. Shevchenko ad, N. Shivarov ao, V. Shoutko ${ }^{\text {z }}$, E. Shumilov ${ }^{\text {, }}$, A. Shvorob ${ }^{\text {ad }}$, T. Siedenburg ${ }^{\text {a }}$, D. Son ${ }^{\text {ap }}$, B. Smith ${ }^{\text {af }}$, P. Spillantini ${ }^{\mathrm{p}}$, M. Steuer ${ }^{\mathrm{n}}$, D.P. Stickland ${ }^{\mathrm{ag}}$, A. Stone ${ }^{\mathrm{f}}$, B. Stoyanov ${ }^{\mathrm{a}}$, A. Straessner ${ }^{\mathrm{a}}$, K. Sudhakar ${ }^{\mathrm{j}}$, G. Sultanov ${ }^{\mathrm{r}}$, L.Z. Sun ${ }^{\mathrm{t}}$, S. Sushkov ${ }^{\text {h}}$, H. Suter ${ }^{\text {av }}$, J.D. Swain ${ }^{\text {r }}$, Z. Szillasi ${ }^{\text {aq, }}$, T. Sztaricskai ${ }^{\text {aq, }, 3}$, X.W. Tang ${ }^{\mathrm{g}}$, L. Tauscher ${ }^{\mathrm{e}}$, L. Taylor ${ }^{\mathrm{k}}$, B. Tellili ${ }^{\mathrm{w}}$, C. Timmermans ${ }^{\mathrm{ac}}$, Samuel C.C. Ting ${ }^{\mathrm{n}}$, S.M. Ting ${ }^{\mathrm{n}}$, S.C. Tonwar ${ }^{\mathrm{j}}$, J. Tóth ${ }^{\mathrm{m}}$, C. Tully ${ }^{\mathrm{q}}$, K.L. Tung ${ }^{\mathrm{g}}$, Y. Uchida ${ }^{\mathrm{n}}$, J. Ulbricht ${ }^{\text {av }}$, E. Valente ${ }^{\text {ah }}$, G. Vesztergombi ${ }^{\mathrm{m}}$, I. Vetlitsky ${ }^{\mathrm{z}}$, D. Vicinanza ${ }^{\text {al }}$, G. Viertel ${ }^{\text {av }}$, S. Villa ${ }^{\mathrm{k}}$, M. Vivargent ${ }^{\mathrm{d}}$, S. Vlachos ${ }^{\mathrm{e}}$, I. Vodopianov ${ }^{\text {ai }}, \mathrm{H}$. Vogel ${ }^{\text {af }}, \mathrm{H}$. Vogt ${ }^{\text {au }}, \mathrm{I}_{\text {. Vorobiev }}{ }^{\text {af }}$, A.A. Vorobyov ai, A. Vorvolakos ${ }^{\mathrm{ab}}, \mathrm{M}$. Wadhwa ${ }^{\mathrm{e}}, \mathrm{W}$. Wallraff ${ }^{\mathrm{a}}, \mathrm{M}$. Wang ${ }^{\mathrm{n}}$, X.L. Wang ${ }^{\mathrm{t}}$, Z.M. Wang ${ }^{\mathrm{t}}$, A. Weber ${ }^{\mathrm{a}}$, M. Weber ${ }^{\mathrm{a}}$, P. Wienemann ${ }^{\mathrm{a}}$, H. Wilkens ${ }^{\mathrm{ac}}$, 
S.X. Wu ${ }^{\text {n}}$, S. Wynhoff ${ }^{\mathrm{a}}$, L. Xia ${ }^{\text {ad }}$, Z.Z. Xu ${ }^{\mathrm{t}}$, J. Yamamoto ${ }^{\mathrm{c}}$, B.Z. Yang ${ }^{\mathrm{t}}$, C.G. Yang ${ }^{\mathrm{g}}$, H.J. Yang ${ }^{\mathrm{g}}$, M. Yang ${ }^{\mathrm{g}}$, J.B. Ye ${ }^{\mathrm{t}}$, S.C. Yeh ${ }^{\text {ay }}$, An. Zalite ${ }^{\text {ai }}$, Yu. Zalite ${ }^{\text {ai }}$, Z.P. Zhang ${ }^{\mathrm{t}}$, G.Y. Zhu ${ }^{\mathrm{g}}$, R.Y. Zhu ${ }^{\text {ad }}$, A. Zichichi ${ }^{\mathrm{i}, \mathrm{q}, \mathrm{r}}$, G. Zilizi $^{\text {aq,3 }}{ }^{\text {, B. Zimmermann }}{ }^{\text {av }}$, M. Zöller ${ }^{\mathrm{a}}$

\author{
a I. Physikalisches Institut, RWTH, D-52056 Aachen, Germany \\ III. Physikalisches Institut, RWTH, D-52056 Aachen, Germany 1 \\ b National Institute for High Energy Physics, NIKHEF, and University of Amsterdam, NL-1009 DB Amsterdam, The Netherlands \\ ${ }^{\mathrm{c}}$ University of Michigan, Ann Arbor, MI 48109, USA \\ d Laboratoire d'Annecy-le-Vieux de Physique des Particules, LAPP, IN2P3-CNRS, BP 110, F-74941 Annecy-le-Vieux CEDEX, France \\ e Institute of Physics, University of Basel, CH-4056 Basel, Switzerland \\ ${ }^{\mathrm{f}}$ Louisiana State University, Baton Rouge, LA 70803, USA \\ $\mathrm{g}$ Institute of High Energy Physics, IHEP, 100039 Beijing, China ${ }^{7}$ \\ ${ }^{\mathrm{h}}$ Humboldt University, D-10099 Berlin, Germany ${ }^{1}$ \\ ${ }^{\mathrm{i}}$ University of Bologna and INFN-Sezione di Bologna, I-40126 Bologna, Italy \\ $\mathrm{j}$ Tata Institute of Fundamental Research, Bombay 400 005, India \\ ${ }^{\mathrm{k}}$ Northeastern University, Boston, MA 02115, USA \\ ${ }^{1}$ Institute of Atomic Physics and University of Bucharest, R-76900 Bucharest, Romania \\ ${ }^{\mathrm{m}}$ Central Research Institute for Physics of the Hungarian Academy of Sciences, H-1525 Budapest 114, Hungary ${ }^{2}$ \\ ${ }^{\mathrm{n}}$ Massachusetts Institute of Technology, Cambridge, MA 02139, USA \\ ${ }^{\circ}$ KLTE-ATOMKI, H-4010 Debrecen, Hungary ${ }^{3}$ \\ $\mathrm{p}$ INFN Sezione di Firenze and University of Florence, I-50125 Florence, Italy \\ q European Laboratory for Particle Physics, CERN, CH-1211 Geneva 23, Switzerland \\ ${ }^{\mathrm{r}}$ World Laboratory, FBLJA Project, CH-1211 Geneva 23, Switzerland \\ ${ }^{\mathrm{s}}$ University of Geneva, CH-1211 Geneva 4, Switzerland \\ ${ }^{\mathrm{t}}$ Chinese University of Science and Technology, USTC, Hefei, Anhui 230 029, China ${ }^{7}$ \\ u University of Lausanne, CH-1015 Lausanne, Switzerland \\ ${ }^{\vee}$ INFN-Sezione di Lecce and Università Degli Studi di Lecce, I-73100 Lecce, Italy \\ ${ }^{\mathrm{w}}$ Institut de Physique Nucléaire de Lyon, IN2P3-CNRS, Université Claude Bernard, F-69622 Villeurbanne, France \\ ${ }^{\mathrm{x}}$ Centro de Investigaciones Energéticas, Medioambientales y Tecnologícas, CIEMAT, E-28040 Madrid, Spain ${ }^{4}$ \\ y INFN-Sezione di Milano, I-20133 Milan, Italy \\ ${ }^{\mathrm{z}}$ Institute of Theoretical and Experimental Physics, ITEP, Moscow, Russia \\ aa INFN-Sezione di Napoli and University of Naples, I-80125 Naples, Italy \\ ${ }^{\mathrm{ab}}$ Department of Natural Sciences, University of Cyprus, Nicosia, Cyprus \\ ${ }^{\text {ac }}$ University of Nijmegen and NIKHEF, NL-6525 ED Nijmegen, The Netherlands \\ ad California Institute of Technology, Pasadena, CA 91125, USA \\ ae INFN-Sezione di Perugia and Università Degli Studi di Perugia, I-06100 Perugia, Italy \\ af Carnegie Mellon University, Pittsburgh, PA 15213, USA \\ ag Princeton University, Princeton, NJ 08544, USA \\ ah INFN-Sezione di Roma and University of Rome, La Sapienza, I-00185 Rome, Italy \\ ai Nuclear Physics Institute, St. Petersburg, Russia \\ aj INFN-Sezione di Napoli and University of Potenza, I-85100 Potenza, Italy \\ ${ }^{\text {ak }}$ University of California, Riverside, CA 92521, USA \\ al University and INFN, Salerno, I-84100 Salerno, Italy \\ am University of California, San Diego, CA 92093, USA
}

an Dept. de Fisica de Particulas Elementales, Univ. de Santiago, E-15706 Santiago de Compostela, Spain

ao Bulgarian Academy of Sciences, Central Lab. of Mechatronics and Instrumentation, BU-1113 Sofia, Bulgaria

${ }^{\mathrm{ap}}$ Laboratory of High Energy Physics, Kyungpook National University, 702-701 Taegu, South Korea

aq University of Alabama, Tuscaloosa, AL 35486, USA

${ }^{a r}$ Utrecht University and NIKHEF, NL-3584 CB Utrecht, The Netherlands

as Purdue University, West Lafayette, IN 47907, USA

at Paul Scherrer Institut, PSI, CH-5232 Villigen, Switzerland au DESY, D-15738 Zeuthen, Germany

av Eidgenössische Technische Hochschule, ETH Zürich, CH-8093 Zürich, Switzerland

aw University of Hamburg, D-22761 Hamburg, Germany

${ }^{a x}$ National Central University, Chung-Li, Taiwan, ROC

ay Department of Physics, National Tsing Hua University, Taiwan, ROC 


\begin{abstract}
Excited leptons are searched for using the L3 detector at LEP. The data collected at centre-of-mass energies in the range from 192 up to $202 \mathrm{GeV}$ correspond to a total luminosity of $233 \mathrm{pb}^{-1}$. No evidence of either pair production of excited leptons, nor of single production is found. From the searches for pair produced excited leptons, lower mass limits close to the kinematic limit are set. From the searches for singly produced excited leptons, upper limits on their couplings are derived in the mass range up to $200 \mathrm{GeV}$. () 2001 Published by Elsevier Science B.V.
\end{abstract}

\section{Introduction}

Several fundamental aspects of the Standard Model [1], such as the number of families and the fermion masses could be explained naturally in composite models where leptons and quarks have substructure [2-4]. The existence of excited leptons is a typical consequence of these models.

The excited leptons $\mathrm{e}^{*}, \mu^{*}, \tau^{*}, \nu_{\mathrm{e}}^{*}, \nu_{\mu}^{*}$ and $\nu_{\tau}^{*}$ were extensively searched for at the LEP $[5,6] \mathrm{e}^{+} \mathrm{e}^{-}$ collider. First generation excited leptons were also searched for at the HERA [7] ep collider. The increase of the LEP centre-of-mass energy, $\sqrt{s}$, up to $202 \mathrm{GeV}$ in 1999 extends the range of potential discovery of new heavy particles.

The interactions of excited leptons are studied within a model [3] that assumes spin 1/2, and isospin

\footnotetext{
1 Supported by the German Bundesministerium für Bildung, Wissenschaft, Forschung und Technologie.

2 Supported by the Hungarian OTKA fund under contract numbers T019181, F023259 and T024011.

3 Also supported by the Hungarian OTKA fund under contract numbers T22238 and T026178.

4 Supported also by the Comisión Interministerial de Ciencia y Tecnología.

5 Also supported by CONICET and Universidad Nacional de La Plata, CC 67, 1900 La Plata, Argentina.

6 Also supported by Panjab University, Chandigarh-160014, India.

7 Supported by the National Natural Science Foundation of China.
}

doublets with left and right handed components:

$$
\mathrm{L}^{*}=\left(\begin{array}{l}
v^{*} \\
\ell^{*}
\end{array}\right)_{L}+\left(\begin{array}{l}
v^{*} \\
\ell^{*}
\end{array}\right)_{R},
$$

with $\ell=\mathrm{e}, \mu, \tau$ and $\nu=v_{\mathrm{e}}, v_{\mu}, v_{\tau}$. Excited leptons are expected to decay into their ground states by radiating a photon $\left(\ell^{*} \rightarrow \ell \gamma, v^{*} \rightarrow v \gamma\right)$, via chargedcurrent decay $\left(\ell^{*} \rightarrow v \mathrm{~W}, v^{*} \rightarrow \ell \mathrm{W}\right)$ or via weak neutral-current decay $\left(\ell^{*} \rightarrow \ell Z, v^{*} \rightarrow v Z\right)$.

At $\mathrm{e}^{+} \mathrm{e}^{-}$colliders, excited leptons can be produced either in pairs $\left(\mathrm{e}^{+} \mathrm{e}^{-} \rightarrow \ell^{*} \ell^{*}, \mathrm{e}^{+} \mathrm{e}^{-} \rightarrow v^{*} v^{*}\right)$ or singly $\left(\mathrm{e}^{+} \mathrm{e}^{-} \rightarrow \ell \ell^{*}, \mathrm{e}^{+} \mathrm{e}^{-} \rightarrow v v^{*}\right)$. In pair production, the coupling of the excited leptons to the gauge bosons is described by the effective Lagrangian:

$\mathcal{L}_{\mathrm{L}^{*} \mathrm{~L}^{*}}=\overline{\mathrm{L}}^{*} \gamma^{\mu}\left(g \frac{\vec{\tau}}{2} \vec{W}_{\mu}+g^{\prime} Y B_{\mu}\right) \mathrm{L}^{*}$.

Here $\gamma^{\mu}$ are the Dirac matrices, $g$ and $g^{\prime}$ are the Standard Model SU(2) and U(1) coupling constants, $\vec{\tau}$ denotes the Pauli matrices, $Y=-1 / 2$ is the hypercharge, and $\vec{W}$ and $B$ are the gauge fields associated with the $\mathrm{SU}(2)$, and $\mathrm{U}(1)$ groups, respectively. The couplings of excited leptons to the gauge bosons are fixed by their quantum numbers, and thus the cross section for pair production depends only on the mass of the excited lepton and the centre-of-mass energy.

The single production of excited leptons as well as their decay are described by means of an effective Lagrangian of the form:

$$
\begin{aligned}
\mathcal{L}_{\mathrm{L} * \mathrm{~L}}= & \frac{1}{\Lambda} \overline{\mathrm{L}}^{*} \sigma^{\mu \nu}\left(g f \frac{\vec{\tau}}{2} \partial_{\mu} \vec{W}_{\nu}+g^{\prime} f^{\prime} Y \partial_{\mu} B_{v}\right) \\
& \times \frac{1-\gamma^{5}}{2} \mathrm{~L}+\text { h.c. },
\end{aligned}
$$


Table 1

Predicted branching ratios for charged and neutral excited lepton decays, for different choices of masses and couplings

\begin{tabular}{|c|c|c|c|c|}
\hline \multirow[t]{3}{*}{ Decay channel } & \multicolumn{4}{|c|}{ Branching ratios } \\
\hline & \multicolumn{2}{|c|}{$M=95 \mathrm{GeV}$} & \multicolumn{2}{|c|}{$M=190 \mathrm{GeV}$} \\
\hline & $f=f^{\prime}$ & $f=-f^{\prime}$ & $f=f^{\prime}$ & $f=-f^{\prime}$ \\
\hline$\ell^{*} \rightarrow \ell \gamma$ & $81 \%$ & - & $36 \%$ & - \\
\hline$\ell^{*} \rightarrow v \mathrm{~W}$ & $19 \%$ & $93 \%$ & $55 \%$ & $63 \%$ \\
\hline$\ell^{*} \rightarrow \ell Z$ & $0 \%$ & $7 \%$ & $9 \%$ & $37 \%$ \\
\hline$v^{*} \rightarrow v \gamma$ & - & $81 \%$ & - & $36 \%$ \\
\hline$v^{*} \rightarrow \ell \mathrm{W}$ & $93 \%$ & $19 \%$ & $63 \%$ & $55 \%$ \\
\hline$v^{*} \rightarrow v Z$ & $7 \%$ & $0 \%$ & $37 \%$ & $9 \%$ \\
\hline
\end{tabular}

where $\sigma^{\mu \nu}=\frac{i}{2}\left[\gamma^{\mu}, \gamma^{\nu}\right], \Lambda$ is the scale of new physics and $f$ and $f^{\prime}$ are the couplings associated with $\mathrm{SU}(2)$ and $\mathrm{U}(1)$, respectively. The production rate of single excited leptons depends on their mass, $\sqrt{s}$, $f / \Lambda$ and $f^{\prime} / \Lambda$. For a given mass and centre-of-mass energy, the polar angle distribution is determined by the relative value of the couplings $f$ and $f^{\prime}$. The decay fractions of excited leptons into standard leptons plus gauge bosons are also determined by the relative value of $f$ and $f^{\prime}$ [4]. Table 1 shows the branching ratios for two choices of $f$ and $f^{\prime}$ and for different excited lepton masses.

Pair production searches are sensitive to excited leptons of mass up to values close to the kinematic limit, i.e., the beam energy, $E_{\text {beam }}$. Searches for single production extend the sensitivity to the mass range above the beam energy up to the centre-of-mass energy.

In the case of single production of excited electrons, the polar angle distribution depends critically on the relative values of $f$ and $f^{\prime}$. If $f=f^{\prime}$, the $t$-channel photon exchange gives a large contribution at low polar angle with the electron escaping through the beam pipe. If $f=-f^{\prime}$, the $\mathrm{e}^{*} \mathrm{e} \gamma$ vertex is not allowed, and only the $\mathrm{Z}$ mediated $s$ - and $t$-channels contribute, giving rise to events with an electron visible in the detector. Thus, the efficiency for $\mathrm{e}^{*}$ selection is calculated for both scenarios: $f=f^{\prime}$ and $f=-f^{\prime}$. For all other flavours of excited leptons, the detection efficiency depends only slightly on the coupling assumption.

\section{Data sample and event simulation}

The data sample analysed was collected in 1999 with the L3 detector [8] at four different centre-ofmass energies, $191.6 \mathrm{GeV}, 195.5 \mathrm{GeV}, 199.5 \mathrm{GeV}$ and $201.7 \mathrm{GeV}$, with corresponding integrated luminosities of $29.7 \mathrm{pb}^{-1}, 83.7 \mathrm{pb}^{-1}, 82.8 \mathrm{pb}^{-1}$ and $37.0 \mathrm{pb}^{-1}$.

Standard Model processes are simulated by different Monte Carlo programs. Radiative Bhabha events are generated using BHWIDE [9] and TEEGG [10]. For other radiative dilepton events, $\mu \mu \gamma, \tau \tau \gamma$ and $v v \gamma$, the KORALZ [11] generator is used. KK2F [12] is used for $\mathrm{q} \overline{\mathrm{q}}(\gamma)$ production. The GGG [13] Monte Carlo is used for final states with only photons. Four-fermion processes are generated with EXCALIBUR [14] for qāev, $\ell \ell \ell \ell$ and $\ell \ell v v$ final states. PYTHIA [15] is used for final states coming from $\mathrm{Z}$ pair and Zee production not covered with EXCALIBUR. Further final states from $\mathrm{W}$ pair production are modelled with KORALW [16]. The production of hadrons in two-photon interactions is described by PHOJET [17] while DIAG36 [18] is used to model lepton production.

Several samples for different flavours and masses of excited leptons are generated in order to optimise the selections and estimate the efficiencies. Pair produced excited leptons are generated with a mass of $95 \mathrm{GeV}$ which is close to the expected limits. In the case of single production, excited leptons with masses 100, 150 and $195 \mathrm{GeV}$ are generated, and a linear interpolation is used to estimate the selection efficiency for masses 
in the range from 90 to $200 \mathrm{GeV}$. The expected differential cross sections [3] and decay modes [4] are modelled. Initial state radiation is not implemented in the generation, but is taken into account in cross section calculations.

The L3 detector response is simulated for all Monte Carlo samples using the GEANT program [19], which takes into account the effects of energy loss, multiple scattering and showering in the detector. Time dependent inefficiencies, as monitored during the data taking period, are reproduced in these simulations.

\section{Selection strategy}

The selection of candidate events for excited lepton production is performed with criteria similar to those used at $\sqrt{s}=189 \mathrm{GeV}$ [5], for the most significant experimental signatures. These criteria are modified to follow the evolution of the signal topology, that manifests a larger boost of the decay particles with the higher $\sqrt{s}$. In addition, some new selections are performed to complement the search in decay channels which were not investigated previously. All selections are based on the identification of the decay products of the excited leptons.

Photons and electrons are identified as clusters with energy above $1 \mathrm{GeV}$ in the BGO electromagnetic calorimeter. Photons must be isolated whereas electrons must be associated to a charged track. In order to be well inside the angular acceptance of the central tracking chamber, the polar angle of electrons and photons must satisfy $|\cos \theta|<0.95$.

Muons are reconstructed either from tracks in the muon spectrometer pointing to the interaction vertex and in time with the event, or via energy depositions in the calorimeters consistent with a minimum ionising particle.

Tau leptons are reconstructed from low multiplicity hadronic jets, or from identified electrons or muons. The neutrino from the tau decay carries away part of the energy, giving rise to events with missing energy along the tau direction. In some selections where a kinematic fit is applied, this signature is imposed by requiring the fitted energy of the tau to be greater than the visible energy of the corresponding jet or lepton.

To reduce the background from the radiative return to the $\mathrm{Z}$ and from two-photon interactions, the missing momentum is required to point away from the beam axis in most of the selections.

The discovery potential is maximised for the topologies in which it is possible to reconstruct the invariant mass of the excited leptons. Pair production and single production of excited leptons are discussed below.

\section{Pair production}

The search for pair produced excited leptons is optimised for excited leptons of masses close to the kinematic limit. For excited leptons of mass up to $100 \mathrm{GeV}$, the neutral-current decay branching ratio is always below $15 \%$, and depends on the mass and the relative value of $f$ and $f^{\prime}$. Thus, only the radiative and the charged-current decays are considered. The production cross section can reach a few $\mathrm{pb}$, depending on the excited lepton mass. For example at $\sqrt{s}=200 \mathrm{GeV}$ and an excited lepton mass of $95 \mathrm{GeV}$, the cross section for pair production is 1.1 $\mathrm{pb}$ for $\ell^{*}$ and $0.7 \mathrm{pb}$ for $v^{*}$.

Table 2 lists the selections and summarises the efficiencies and the number of events found in data and expected from Standard Model background processes. The relative importance of the selections is given by the coupling assumption.

\subsection{Radiative decays}

The radiative decays $\ell^{*} \rightarrow \ell \gamma$ and $\nu^{*} \rightarrow \nu \gamma$ provide the maximum sensitivity in the $f=f^{\prime}$ and the $f=-f^{\prime}$ scenarios respectively. Event selection for the $\mathrm{e}^{+} \mathrm{e}^{-} \rightarrow \ell^{*} \ell^{*} \rightarrow \ell \ell \gamma \gamma$ process requires low multiplicity events with two photons and two same flavour charged leptons. To reduce the background from radiative dilepton production, at least one of the photons is required to lie in the central region of the detector $\left(\left|\cos \theta_{\gamma}\right|<0.75\right.$, where $\theta_{\gamma}$ is the photon polar angle). After a kinematic fit, which imposes energy and momentum conservation, the difference of the leptonphoton invariant masses is required to be smaller than $10 \mathrm{GeV}$ and their sum greater than $100 \mathrm{GeV}$.

The selection of the $\mathrm{e}^{+} \mathrm{e}^{-} \rightarrow v^{*} v^{*} \rightarrow \nu v \gamma \gamma$ channel requires two photons in the central region of the detector with energies $E_{\gamma}$ in the range $0.2<$ $E_{\gamma} / E_{\text {beam }}<0.8$. There should be no tracks in the central tracker nor in the muon spectrometer, and the en- 
Table 2

Number of candidates $N_{D}$, number of background events $N_{B}$, and average signal efficiencies $\epsilon$, in the pair production (upper part) and the single production (lower part) searches

\begin{tabular}{|c|c|c|c|c|c|c|c|c|}
\hline & \multicolumn{4}{|c|}{ Charged excited leptons } & \multicolumn{4}{|c|}{ Excited neutrinos } \\
\hline & Signal & $N_{D}$ & $N_{B}$ & $\epsilon$ & Signal & $N_{D}$ & $N_{B}$ & $\epsilon$ \\
\hline \multirow[t]{7}{*}{ Pair production } & $\mathrm{e}^{*} \mathrm{e}^{*} \rightarrow \mathrm{ee} \gamma \gamma$ & 1 & 0.8 & $46 \%$ & $v_{\mathrm{e}}^{*} v_{\mathrm{e}}^{*} \rightarrow$ eeWW & 0 & 0.2 & $19 \%$ \\
\hline & $\mu^{*} \mu^{*} \rightarrow \mu \mu \gamma \gamma$ & 1 & 0.5 & $44 \%$ & $v_{\mu}^{*} v_{\mu}^{*} \rightarrow \mu \mu \mathrm{WW}$ & 0 & 0.7 & $22 \%$ \\
\hline & $\tau^{*} \tau^{*} \rightarrow \tau \tau \gamma \gamma$ & 0 & 0.2 & $43 \%$ & $\nu_{\tau}^{*} \nu_{\tau}^{*} \rightarrow \tau \tau \mathrm{WW}$ & 2978 & 2993 & $69 \%$ \\
\hline & $\ell^{*} \ell^{*} \rightarrow \nu \nu \mathrm{WW}$ & 2978 & 2993 & $65 \%$ & $v^{*} v^{*} \rightarrow v v \gamma \gamma$ & 2 & 1.9 & $45 \%$ \\
\hline & $\mathrm{e}^{*} \mathrm{e}^{*} \rightarrow \mathrm{e} \gamma \nu \mathrm{W}$ & 13 & 11 & $57 \%$ & $v_{\mathrm{e}}^{*} v_{\mathrm{e}}^{*} \rightarrow v \gamma \mathrm{eW}$ & 18 & 12 & $38 \%$ \\
\hline & $\mu^{*} \mu^{*} \rightarrow \mu \gamma \nu \mathrm{W}$ & 5 & 8 & $43 \%$ & $v_{\mu}^{*} v_{\mu}^{*} \rightarrow v \gamma \mu \mathrm{W}$ & 18 & 12 & $28 \%$ \\
\hline & $\tau^{*} \tau^{*} \rightarrow \tau \gamma \nu \mathrm{W}$ & 56 & 45 & $37 \%$ & $\nu_{\tau}^{*} \nu_{\tau}^{*} \rightarrow \nu \gamma \tau \mathrm{W}$ & 18 & 12 & $19 \%$ \\
\hline \multirow[t]{9}{*}{ Single production } & $\mathrm{ee}^{*} \rightarrow \mathrm{ee} \gamma$ & 682 & 714 & $61 \%$ & $v v^{*} \rightarrow v v \gamma$ & 230 & 232 & $64 \%$ \\
\hline & $\mu \mu^{*} \rightarrow \mu \mu \gamma$ & 72 & 67 & $61 \%$ & & 230 & 232 & $64 \%$ \\
\hline & $\tau \tau^{*} \rightarrow \tau \tau \gamma$ & 50 & 69 & $42 \%$ & & 230 & 232 & $64 \%$ \\
\hline & $\mathrm{ee}^{*} \rightarrow \mathrm{e} v_{\mathrm{e}} \mathrm{W}$ & 728 & 764 & $67 \%$ & $v_{\mathrm{e}} v_{\mathrm{e}}^{*} \rightarrow v_{\mathrm{ee}} \mathrm{W}$ & 728 & 764 & $65 \%$ \\
\hline & $\mu \mu^{*} \rightarrow \mu v_{\mu} \mathrm{W}$ & 684 & 694 & $60 \%$ & $v_{\mu} v_{\mu}^{*} \rightarrow v_{\mu} \mu \mathrm{W}$ & 684 & 694 & $65 \%$ \\
\hline & $\tau \tau^{*} \rightarrow \tau \nu_{\tau} \mathrm{W}$ & 1365 & 1377 & $55 \%$ & $\nu_{\tau} \nu_{\tau}^{*} \rightarrow v_{\tau} \tau \mathrm{W}$ & 1365 & 1377 & $56 \%$ \\
\hline & $\mathrm{ee}^{*} \rightarrow \mathrm{eeZ}$ & 663 & 692 & $58 \%$ & $\nu v^{*} \rightarrow \nu v Z$ & 350 & 357 & $29 \%$ \\
\hline & $\mu \mu^{*} \rightarrow \mu \mu \mathrm{Z}$ & 608 & 617 & $52 \%$ & & 350 & 357 & $29 \%$ \\
\hline & $\tau \tau^{*} \rightarrow \tau \tau \mathrm{Z}$ & 1307 & 1308 & $34 \%$ & & 350 & 357 & $29 \%$ \\
\hline
\end{tabular}

ergy of any other calorimetric cluster should be less than $5 \mathrm{GeV}$. The background from the $\mathrm{e}^{+} \mathrm{e}^{-} \rightarrow \gamma \gamma(\gamma)$ process is efficiently reduced by requiring the photon acoplanarity to be greater than $10^{\circ}$.

\subsection{Charged-current decays}

The charged-current decays $\ell^{*} \rightarrow \nu \mathrm{W}$ and $v^{*} \rightarrow$ $\ell \mathrm{W}$ are dominant in the $f=-f^{\prime}$ and the $f=f^{\prime}$ scenarios, respectively. Final states from $\mathrm{e}^{+} \mathrm{e}^{-} \rightarrow$ $\ell^{*} \ell^{*} \rightarrow \nu \nu \mathrm{WW}$ are experimentally similar to Standard Model W pair production. To achieve a high signal efficiency, pair production of excited leptons is searched for as an enhancement of the $\mathrm{W}$ pair-like event rate. A combination of four selections, named qqqq, qqev, $\mathrm{qq} \mu \nu$ and $\mathrm{qq} \tau \nu$, selects such events.
For the qqqq selection, events with at least three hadronic jets and visible energy greater than $140 \mathrm{GeV}$ are selected. For at least one pair of jets, their invariant mass must be in the range between $50 \mathrm{GeV}$ and $110 \mathrm{GeV}$, the recoiling mass against these two jets must be greater than $50 \mathrm{GeV}$ and the sum of invariant plus recoil masses must be greater than $120 \mathrm{GeV}$. Fig. 1(a) shows the sum of invariant and recoil masses for this pair of jets. The signal distribution corresponds to the $f=-f^{\prime}$ coupling assumption.

For the $\mathrm{qq} \ell v$ selections, high multiplicity events with missing momentum greater than $20 \mathrm{GeV}$, visible energy below $0.8 \sqrt{s}$ and an isolated lepton are retained. The invariant mass of the hadronic system must be compatible with the $\mathrm{W}$ mass. In the qq $\tau \nu$ selection, the $\mathrm{q} \overline{\mathrm{q}}(\gamma)$ background is further reduced by 

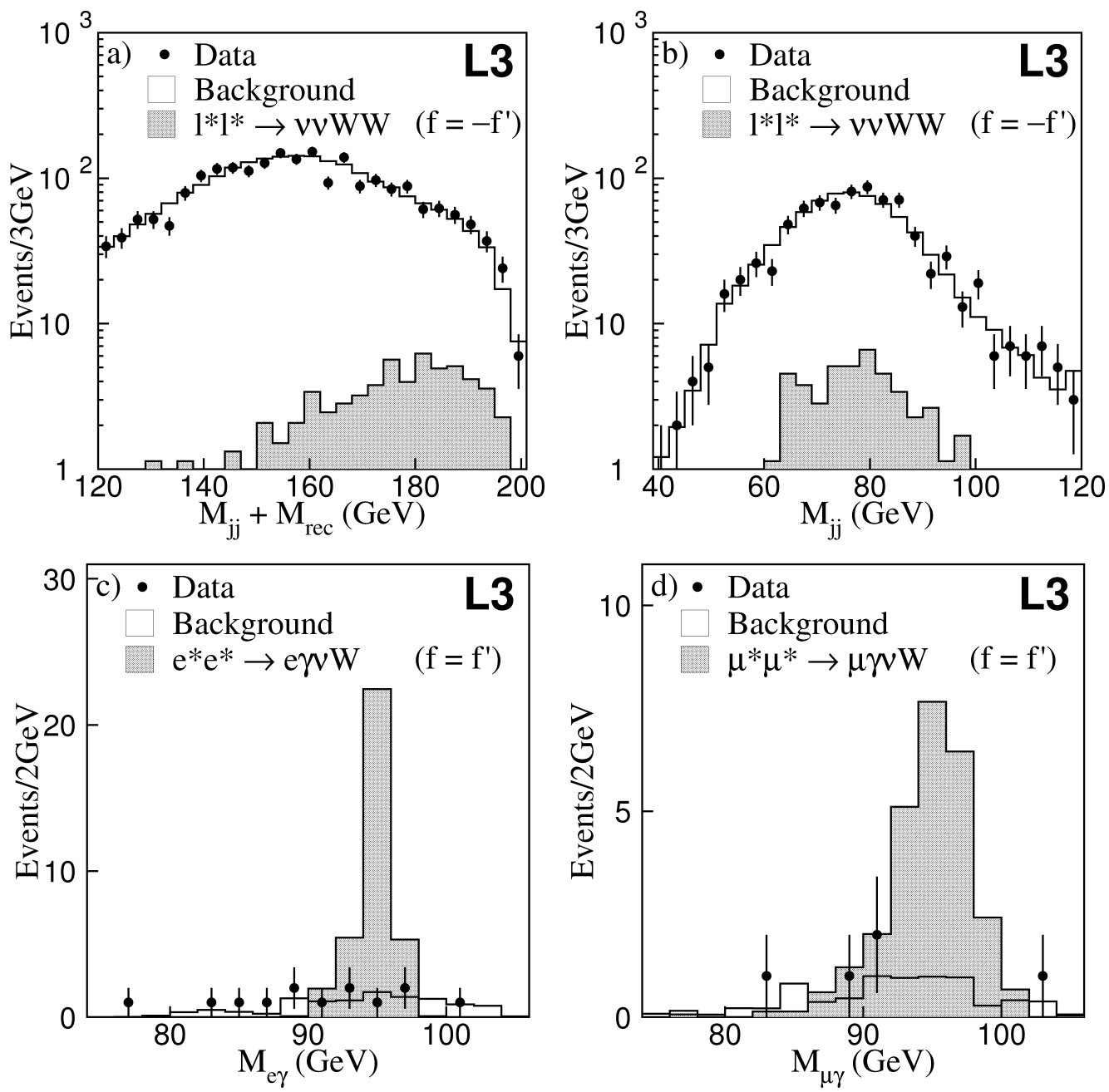

Fig. 1. Distributions of (a) the sum of invariant, $M_{j j}$, and recoil mass, $M_{\mathrm{rec}}$, for two jets in the qqqq selection; (b) the hadronic invariant mass, $M_{j j}$, in the qq $\ell v$ selection; (c) the electron photon invariant mass in the e $\gamma \nu \mathrm{W}$ selection and (d) the muon photon invariant mass in the $\mu \gamma \nu \mathrm{W}$ selection. The expected signal for an excited lepton with a mass of $95 \mathrm{GeV}$ is shown together with data and Standard Model background; its luminosity averaged cross section is $0.93 \mathrm{pb}$.

requiring the missing momentum along the longitudinal direction to be smaller than $55 \mathrm{GeV}$, and rejecting events with high energy photons. Fig. 1(b) shows the hadronic invariant mass for the qq $\ell v$ selected events.

The experimental signatures of the process $\mathrm{e}^{+} \mathrm{e}^{-} \rightarrow$ $\nu^{*} \nu^{*} \rightarrow \ell \ell \mathrm{WW}$ are also similar to Standard Model W pair production. Thus, the qqqq and qq $\ell v$ selections described above are used to investigate them. Moreover, in the eeWW and $\mu \mu \mathrm{WW}$ channels, the final state leptons may be detected, providing a very clean signature. Therefore, for these channels the signal sensitivity is enhanced by requiring two additional isolated electrons or muons in the event, with energy in the range between $3 \mathrm{GeV}$ and $25 \mathrm{GeV}$.

\subsection{Mixed decays}

The processes $\mathrm{e}^{+} \mathrm{e}^{-} \rightarrow \ell^{*} \ell^{*} \rightarrow \ell \gamma \nu \mathrm{W}$ and $\mathrm{e}^{+} \mathrm{e}^{-} \rightarrow$ $\nu^{*} \nu^{*} \rightarrow v \gamma \ell \mathrm{W}$ give rise to events with an energetic photon, a lepton, and the decay products of a $\mathrm{W}$ bo- 
son. Both the hadronic and the leptonic decays of the $\mathrm{W}$ are considered. The selected events must contain an energetic photon at high polar angle. The additional criteria for each final state are discussed below.

In the $\mathrm{e}^{*} \mathrm{e}^{*} \rightarrow \mathrm{e} \gamma \nu \mathrm{W}$ and $\mu^{*} \mu^{*} \rightarrow \mu \gamma \nu \mathrm{W}$ channels, an energetic electron or muon is required. Moreover, the sum of the invariant and recoil masses of the lepton-photon pair, i.e., the masses of the $\ell^{*}$ candidates, must be greater than $160 \mathrm{GeV}$ and their difference smaller than $10 \mathrm{GeV}$ in the electron case and $20 \mathrm{GeV}$ in the muon case. Fig. 1(c) and (d) show the $\mathrm{e} \gamma$ and $\mu \gamma$ invariant masses for the selected events. For the signal distributions, $f=f^{\prime}$ is assumed to set the branching ratios.

For the $\tau^{*} \tau^{*} \rightarrow \tau \gamma \nu \mathrm{W}$ and $\nu^{*} \nu^{*} \rightarrow \nu \gamma \ell \mathrm{W}$ channels, a low multiplicity jet, an electron or a muon must be present. In high multiplicity events, the signature of a $\mathrm{W}$ boson is imposed by requiring the invariant mass of the hadronic system to be in the range from $60 \mathrm{GeV}$ to $100 \mathrm{GeV}$, whereas low multiplicity events must contain only the photon and no more than two leptons. For the $\tau^{*} \tau^{*} \rightarrow \tau \gamma \nu \mathrm{W}$ channel, the invariant mass and the energy of the $\nu \mathrm{W}$ system correspond to the $\tau^{*}$ mass and the beam energy, respectively. This signature is used by requiring the recoil mass of the tau-photon system to exceed $80 \mathrm{GeV}$, and the visible energy of the rest of the event to be smaller than $120 \mathrm{GeV}$. For the $v^{*} v^{*} \rightarrow v \gamma \ell \mathrm{W}$ channel, after excluding the photon, the visible energy of the rest of the event must be smaller than $120 \mathrm{GeV}$ and $70 \mathrm{GeV}$ for the hadronic and leptonic decays of the $\mathrm{W}$, respectively.

\section{Single production}

The search for singly produced excited leptons complements the search for pair production in the mass range from $E_{\text {beam }}$ to $\sqrt{s}$. The selections are optimised to cover this wide range of masses with high efficiency. The radiative decays of excited leptons, as well as the charged-current decays and the neutralcurrent decays are investigated, as described below. Both the hadronic and the leptonic decays of $\mathrm{W}$ and $\mathrm{Z}$ bosons are considered.

Table 2 lists the selections and summarises the efficiencies and the number of events found in data and expected from Standard Model background processes.

\subsection{Radiative decays}

The event selection for $\mathrm{e}^{+} \mathrm{e}^{-} \rightarrow \ell \ell^{*} \rightarrow \ell \ell \gamma$ identifies final states with two leptons and one photon with energy greater than $20 \mathrm{GeV}$. The background from radiative dilepton production is reduced by requiring the photon to be in the central region of the detector, $\left|\cos \theta_{\gamma}\right|<0.75$. After a kinematic fit, at least one of the two possible $\ell \gamma$ invariant masses must be greater than $70 \mathrm{GeV}$. Events with just an identified electron and a photon, with invariant mass above $70 \mathrm{GeV}$, are also accepted for the excited electron selection. Thus, a high signal efficiency is kept for the signal events originating from the $t$-channel photon exchange where one electron escapes along the beam pipe. Fig. 2(a)-(c) show all combinations of leptonphoton invariant masses above $90 \mathrm{GeV}$.

Final states from $\mathrm{e}^{+} \mathrm{e}^{-} \rightarrow \nu v^{*} \rightarrow v v \gamma$ are selected by requiring a single photon with energy greater than $0.15 \sqrt{s}$ at high polar angle $\left|\cos \theta_{\gamma}\right|<0.75$. Neither tracks in the tracking chamber, nor in the muon spectrometer should be present. Fig. 2(d) shows the distribution of the normalised photon energy.

\subsection{Charged-current decays}

The experimental signatures of $\mathrm{e}^{+} \mathrm{e}^{-} \rightarrow \ell \ell^{*} \rightarrow$ $\ell \nu \mathrm{W}$ and $\mathrm{e}^{+} \mathrm{e}^{-} \rightarrow \nu \nu^{*} \rightarrow \nu \ell \mathrm{W}$ are similar to $\mathrm{W}$ pair production. For the final states containing a pair of hadronic jets from the $\mathrm{W}$ decay, the qq $\ell v$ selections described above are applied. However, in order to be sensitive to charged excited leptons with mass close to the $\mathrm{W}$ mass, and to excited neutrinos with mass close to $\sqrt{s}$, where the final state neutrino carries low momentum, no cuts on the missing momentum or visible energy are applied. Fig. 3(a)-(c) show the recoil mass against the identified lepton in the qqev, $\mathrm{qq} \mu \nu$ and $\mathrm{qq} \tau \nu$ selections, indicating the mass of the $\ell^{*}$ candidates. Fig. 3(d)-(f) show the event invariant mass for the qqe $v$, qq $\mu \nu$ and qq $\tau \nu$ selected events, indicating the mass of the $v^{*}$ candidates.

For the cases in which the final state lepton escapes undetected, the qq $\ell v$ selections are complemented with a qqvv selection which requires two acoplanar hadronic jets with invariant mass between $60 \mathrm{GeV}$ and $100 \mathrm{GeV}$, visible energy smaller than $150 \mathrm{GeV}$ and recoil mass below $70 \mathrm{GeV}$. 

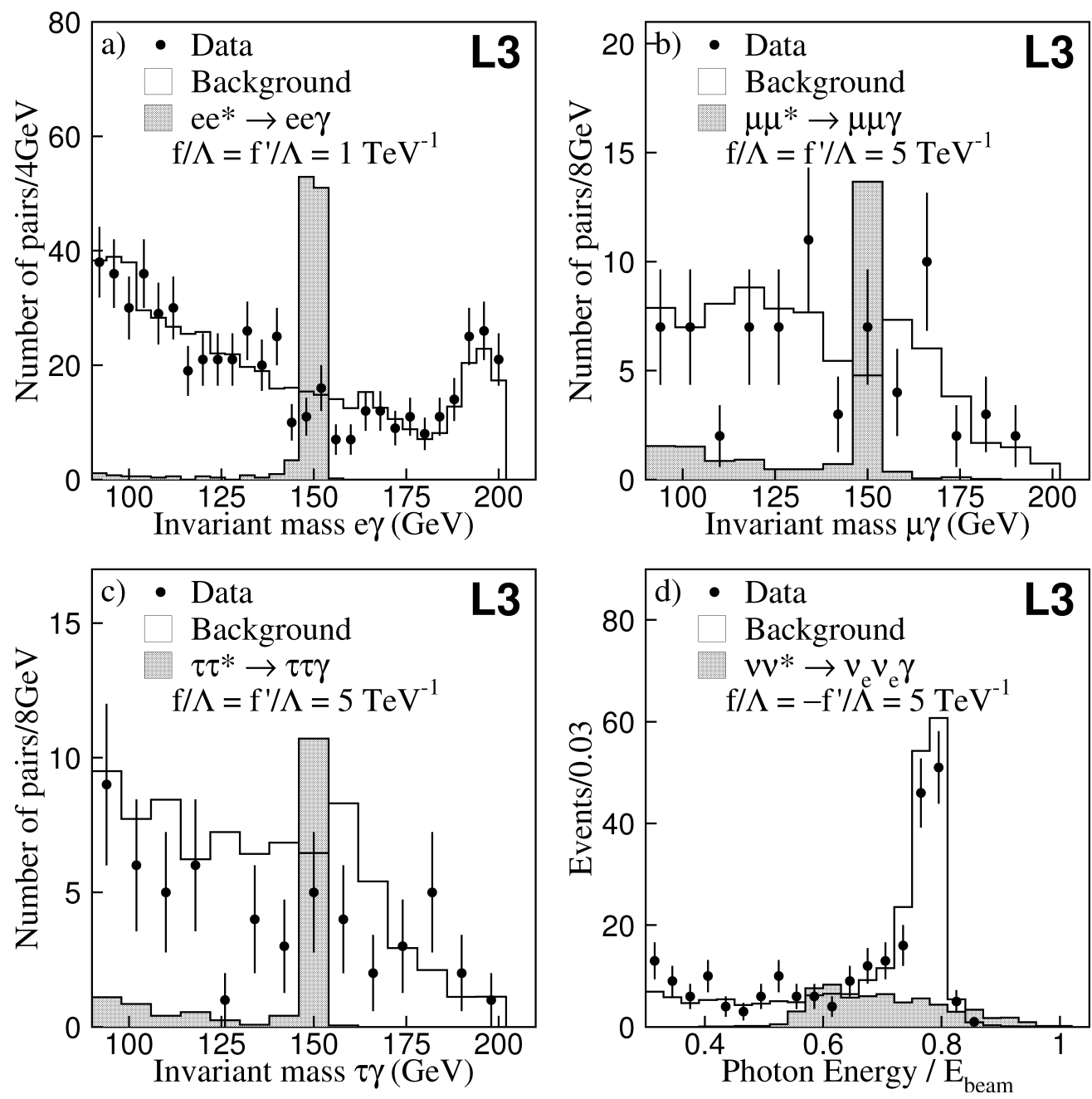

Fig. 2. The invariant mass distributions for (a) $\mathrm{e} \gamma$, (b) $\mu \gamma$, and (c) $\tau \gamma$ pairs. (d) The normalised energy distribution of single photon events. The expected signal for an excited lepton with a mass of $150 \mathrm{GeV}$ is shown together with data and Standard Model background. The signals are plotted for the arbitrary choice of couplings displayed in the figures.

If the $\mathrm{W}$ boson decays leptonically, the event contains two charged leptons and two neutrinos, $\ell \ell^{*} \rightarrow$ $\ell \nu \mathrm{W} \rightarrow \ell \nu \ell \nu$ or $\nu \nu^{*} \rightarrow \nu \ell \mathrm{W} \rightarrow \nu \ell \ell \nu$. For this signature, low multiplicity events containing two charged leptons with acoplanarity between $10^{\circ}$ and $170^{\circ}$ are selected. The energy of the most energetic lepton must exceed $20 \mathrm{GeV}$ and the second lepton must exceed $3 \mathrm{GeV}$. To reject the background from two-photon interactions, the transverse missing momentum must be greater than $10 \mathrm{GeV}$.

\subsection{Neutral-current decays}

The processes $\mathrm{e}^{+} \mathrm{e}^{-} \rightarrow \ell \ell^{*} \rightarrow \ell \ell \mathrm{Z}$ and $\mathrm{e}^{+} \mathrm{e}^{-} \rightarrow$ $\nu v^{*} \rightarrow \nu v Z$ give rise to four visible experimental signatures according to the decay channel of the $Z$ boson: qq $\ell \ell, \mathrm{qq} v v, \ell \ell \ell \ell$ and $\ell \ell v v$. The qq $\ell \ell, \mathrm{qq} v v$ and $\ell \ell v v$ signatures are investigated making use of the same selections used in the search for single excited leptons decaying via charged current. For the $\ell \ell \ell \ell$ final state, low multiplicity events with at least three 

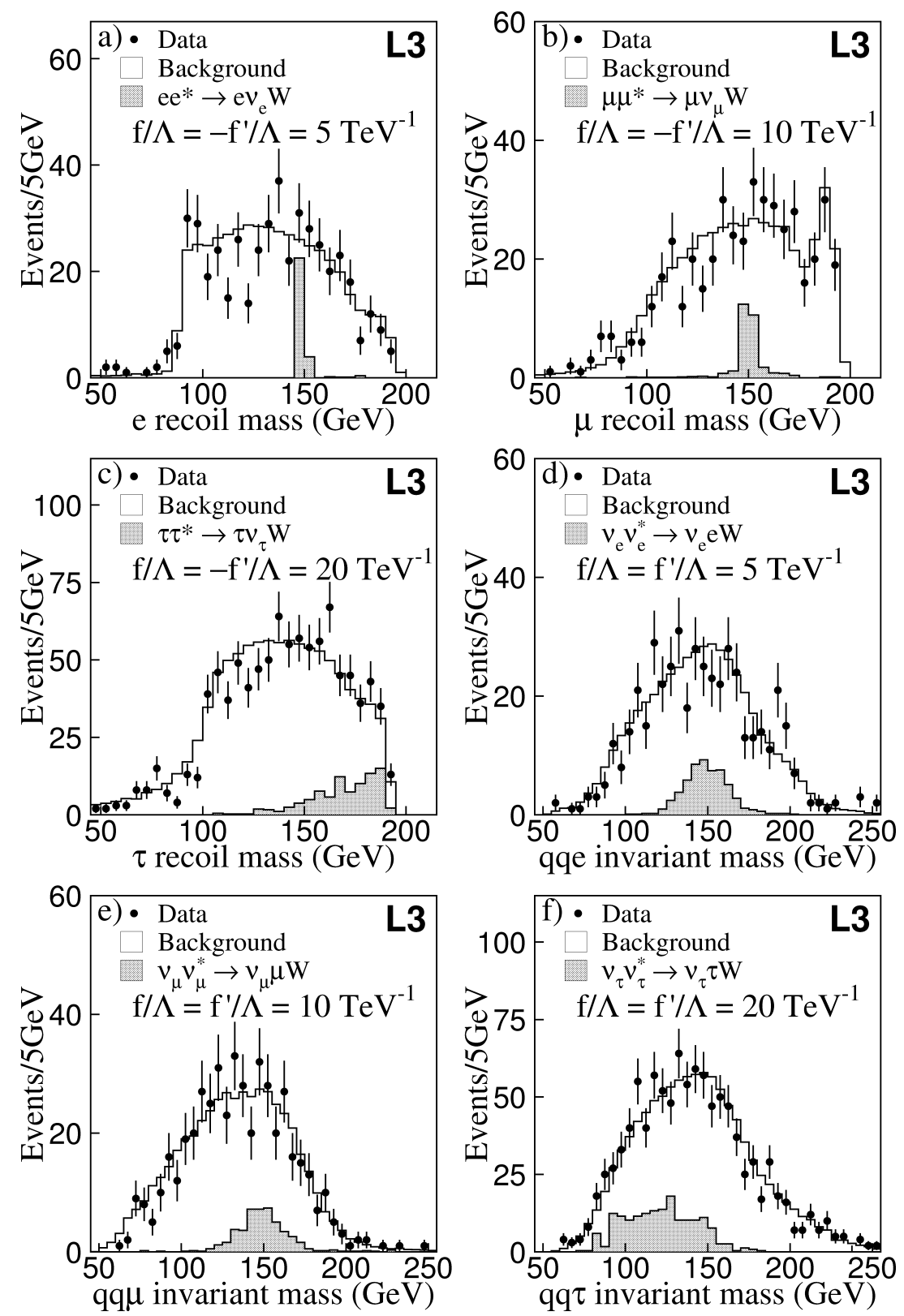

Fig. 3. Lepton recoil mass distributions for the (a) qqev, (b) qq $\mu \nu$ and (c) qq $\tau v$ selections. Invariant mass distributions for (d) qqe $v$, (e) qq $\mu \nu$ and (f) qq $\tau v$ selected events. The expected signal for an excited lepton with a mass of $150 \mathrm{GeV}$ is shown together with data and Standard Model background. The signals are plotted for the arbitrary choice of couplings displayed in the figures. 
charged leptons are selected. The invariant mass of a pair of these leptons must be in the range from $80 \mathrm{GeV}$ to $100 \mathrm{GeV}$.

\section{Results}

The number of events observed in the data for each of the selections described above is consistent with the Standard Model background expectation, as shown in Table 2. For some signatures of excited leptons, a sizable signal efficiency is achieved with very low background, giving sensitivity to production cross sections as low as $0.1 \mathrm{pb}$.

For each flavour of excited lepton, all selections are combined to derive an upper limit to the signal cross section, taking into account the luminosity, the branching fractions and the efficiencies. The results from the four centre-of-mass energies analysed are presented together, but are treated separately for the calculation of limits, as the signal cross sections depend on $\sqrt{s}$. These combinations include the results from $\sqrt{s}=189 \mathrm{GeV}$ [5]. The limit is set at $95 \%$ confidence level, using Bayesian statistics and assuming a flat positive a priori distribution for the signal cross section. Two different scenarios are considered in order to present limits. In the first one, $f=f^{\prime}$, the radiative decay is allowed for charged excited leptons whereas it is forbidden for excited neutrinos. In the second one, $f=-f^{\prime}$, the situation is the opposite: the radiative decay is forbidden for charged excited leptons and is allowed for excited neutrinos.

In the case of pair production, lower mass limits are derived from the cross section upper bounds. A scan is performed for all the possible relative values of $f$ and $f^{\prime}$. For each value, the corresponding decay fractions $\ell^{*} \ell^{*} \rightarrow \ell \ell \gamma \gamma, \ell^{*} \ell^{*} \rightarrow \nu \nu \mathrm{WW}$ and $\ell^{*} \ell^{*} \rightarrow \ell \gamma \nu \mathrm{W}$ or $\nu^{*} \nu^{*} \rightarrow \nu \nu \gamma \gamma, \nu^{*} \nu^{*} \rightarrow \ell \ell \mathrm{WW}$ and $\nu^{*} \nu^{*} \rightarrow \nu \gamma \ell \mathrm{W}$ in the case of excited neutrinos are calculated, and a mass limit is set. In Table 3 the limits corresponding to the $f=f^{\prime}$ and $f=-f^{\prime}$ scenarios are presented together with the lowest limit obtained in the scan. This limit corresponds to the ratio of the couplings $f / f^{\prime}$ that, given the signal efficiencies and the number of expected candidates, maximise the compatibility of the data with the existence of excited leptons. The lowest mass limits correspond to the branching ratios that, given the signal efficiencies
Table 3

$95 \%$ confidence level lower mass limits for the different excited leptons obtained from pair production searches. For each flavour, the mass limits for $f=f^{\prime}, f=-f^{\prime}$ and for the coupling independent case, are shown

\begin{tabular}{cccc}
\hline \multirow{2}{*}{$\begin{array}{c}\text { Excited } \\
\text { lepton }\end{array}$} & \multicolumn{3}{c}{$95 \%$ CL mass limit $(\mathrm{GeV})$} \\
\cline { 2 - 4 } $\mathrm{e}^{*}$ & 100.0 & 93.4 & 93.3 \\
$\mu^{*}$ & 100.2 & 93.4 & 93.4 \\
$\tau^{*}$ & 99.8 & 93.4 & 92.2 \\
& & & Any coupling \\
$v_{\mathrm{e}}^{*}$ & 99.1 & 99.4 & 98.2 \\
$v_{\mu}^{*}$ & 99.3 & 99.4 & 98.3 \\
$v_{\tau}^{*}$ & 90.5 & 99.4 & 87.8 \\
\hline
\end{tabular}

and the number of expected candidates, maximise the compatibility of the data with the existence of excited leptons.

In the case of single production searches, an upper limit to the cross section is obtained as a function of the excited lepton mass. The distributions of the variables presented in Figs. 2 and 3 are investigated for each mass hypothesis. The number of candidates found in data and expected from background, as well as the efficiency are calculated in the range where the signal is expected. For the charged-current decay with the $\mathrm{W}$ decaying leptonically, and for the neutralcurrent decay, all the selected events are considered as candidates for all the mass hypotheses. The results from all selections are combined to derive a cross section limit which is interpreted in terms of an upper limit to the effective coupling constant $|f| / \Lambda$. Fig. 4 shows the upper limits to the effective coupling $|f| / \Lambda$ for charged excited leptons and excited neutrinos for the $f=f^{\prime}$ and $f=-f^{\prime}$ scenarios. The left-hand edge of the curves indicates the lower mass limit derived from pair production searches whereas the rise of the curves in the high excited lepton mass region reflects the lack of experimental sensitivity due to the low expected signal cross section. The limits corresponding to charged excited leptons in the $f=f^{\prime}$ scenario and excited neutrinos in the $f=-f^{\prime}$ scenario are derived mainly from the radiative decay searches, highly sensitive to the signal. These limits are therefore more stringent than the limits obtained in the complementary scenarios in which the radiative decays are forbid- 

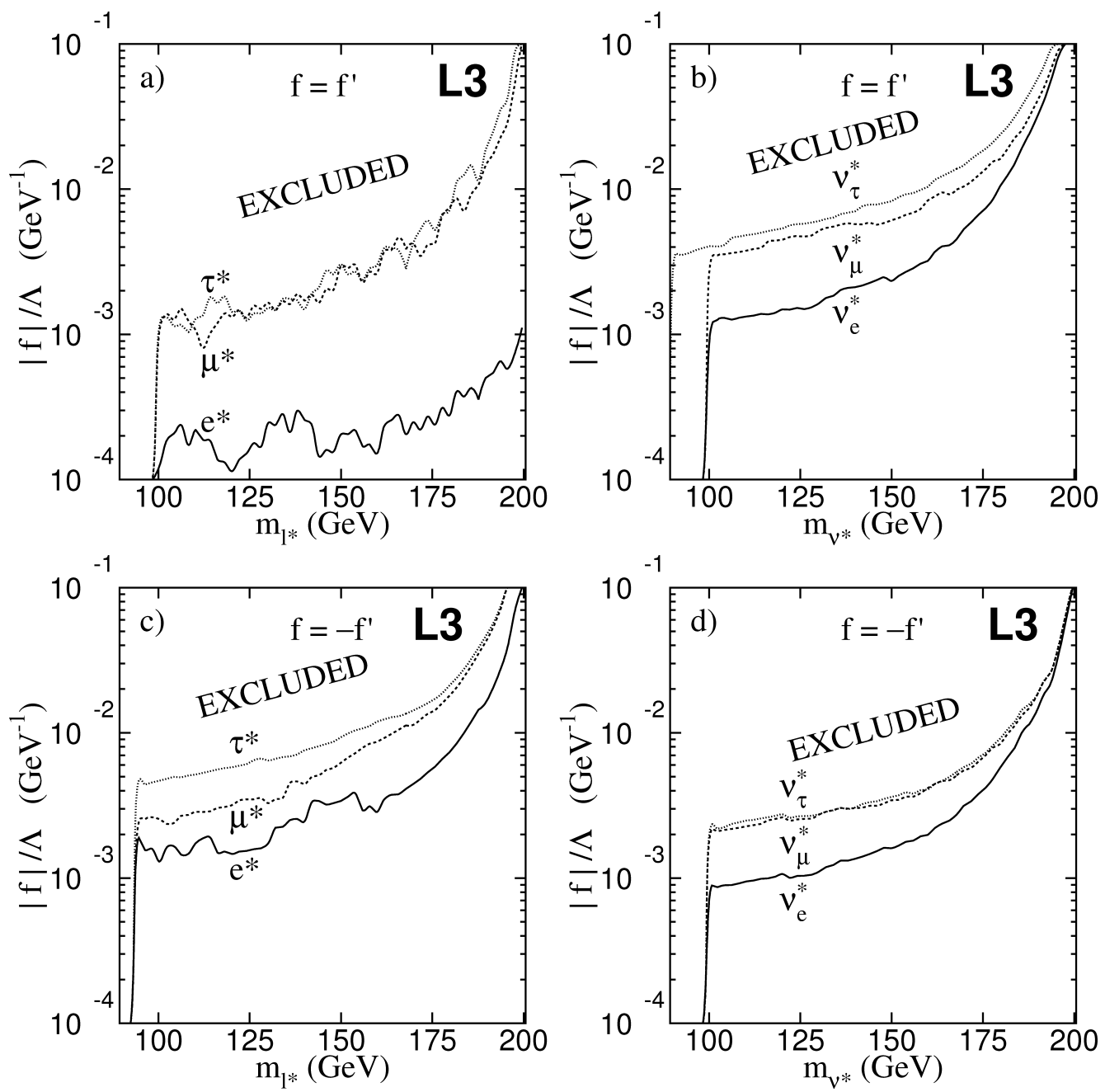

Fig. 4. 95\% confidence level upper limits on the effective coupling $|f| / \Lambda$, as a function of the excited lepton mass with $f=f^{\prime}:\left(\right.$ a) $\mathrm{e}^{*}, \mu^{*}$ and $\tau^{*}$, (b) $v_{\mathrm{e}}^{*}, v_{\mu}^{*}$ and $v_{\tau}^{*}$, and with $f=-f^{\prime}:$ (c) $\mathrm{e}^{*}, \mu^{*}$ and $\tau^{*}$,(d) $v_{\mathrm{e}}^{*}, v_{\mu}^{*}$ and $v_{\tau}^{*}$.

den. Moreover, the limits corresponding to excited leptons of the first generation are significantly lower due to the higher cross section explained by the $t$-channel contribution.

The systematic uncertainties are conservatively taken into account for the limit calculations by lowering the background expectation and the signal efficiency in all the selections. A variation close to $2.6 \%$ in the background, depending on the selection, accounts for the uncertainties related to background cross sections, limited Monte Carlo statistics, detector simulation and selection efficiency. A similar variation in the signal efficiency accounts for the uncertainties coming from signal Monte Carlo statistics, the extrapolation to different centre-of-mass energies and excited lepton masses and detector simulation. The effect of systematics is quite significant for the limits extracted from high statistics selections, as in the case of $v_{\tau}^{*}$ in the $f=f^{\prime}$ scenario.

In conclusion, lower mass limits as high as $98.3 \mathrm{GeV}$ are set for any value of the couplings. Upper limits on the couplings $|f| / \Lambda$ ranging from $10^{-1}$ to 
$10^{-4} \mathrm{GeV}^{-1}$ are derived in the mass range from $90 \mathrm{GeV}$ to $200 \mathrm{GeV}$.

\section{Acknowledgements}

We thank the CERN accelerator divisions for the continuous and successful upgrade of the LEP machine and its excellent performance. We acknowledge the contributions of the engineers and technicians who have participated in the construction and maintenance of this experiment.

\section{References}

[1] S.L. Glashow, Nucl. Phys. 22 (1961) 579;

A. Salam, in: N. Svartholm (Ed.), Elementary Particle Theory, Almqvist and Wiksell, Stockholm, 1968;

S. Weinberg, Phys. Rev. Lett. 19 (1967) 1264.

[2] F. Boudjema et al., in: J. Ellis et al. (Eds.), Z. Physics at LEP 1, Vol. 2, p. 188, Report CERN/89/08 (1989), and references therein.

[3] K. Hagiwara et al., Z. Phys. C 29 (1985) 115.

[4] M.B. Voloshin et al., Sov. Phys. JETP 64 (1986) 446;

M.B. Voloshin, Phys. Lett. B 209 (1988) 360;

F. Boudjema et al., Phys. Lett. B 240 (1990) 485;

F. Boudjema et al., Z. Phys. C 57 (1993) 425.

[5] L3 Collaboration, M. Acciarri et al., Phys. Lett. B 473 (2000) 177.

[6] ALEPH Collaboration, D. Buskulic et al., Phys. Lett. B 385 (1996) 445 ;

DELPHI Collaboration, P. Abreu et al., Eur. Phys. J. C 8 (1999) 41 ;

OPAL Collaboration, G. Abbiendi et al., Eur. Phys. J. C 14 (2000) 73.

[7] H1 Collaboration, S. Aid et al., Nucl. Phys. B 483 (1997) 44;
ZEUS Collaboration, J. Breitweg et al., Z. Phys. C 76 (1997) 631.

[8] L3 Collaboration, B. Adeva et al., Nucl. Instrum. Methods A 289 (1990) 35;

L3 Collaboration, O. Adriani et al., Phys. Rep. 236 (1993) 1;

M. Chemarin et al., Nucl. Instrum. Methods A 349 (1994) 345;

M. Acciarri et al., Nucl. Instrum. Methods A 351 (1994) 300;

G. Basti et al., Nucl. Instrum. Methods A 374 (1996) 293;

A. Adam et al., Nucl. Instrum. Methods A 383 (1996) 342.

[9] BHWIDE version 1.01 is used;

S. Jadach et al., Phys. Rev. D 40 (1989) 3582;

S. Jadach et al., Comput. Phys. Commun. 70 (1992) 305;

S. Jadach et al., Phys. Lett. B 390 (1997) 298.

[10] D. Karlen, Nucl. Phys. B 289 (1987) 23.

[11] KORALZ version 4.02 is used; S. Jadach et al., Comput. Phys. Commun. 79 (1994) 503.

[12] KK2F version 4.12 is used; S. Jadach et al., Comput. Phys. Commun. 130 (2000) 260.

[13] F.A. Berends et al., Nucl. Phys. B 186 (1981) 22; CALKUL Collaboration, F.A. Berends et al., Nucl. Phys. B 239 (1984) 395.

[14] F.A. Berends et al., Comput. Phys. Commun. 85 (1995) 437.

[15] PYTHIA versions 5.722 and 6.1 are used;

T. Sjöstrand, Preprint CERN-TH/7112/93 (1993), revised August 1995;

T. Sjöstrand, Comput. Phys. Commun. 82 (1994) 74;

T. Sjöstrand, hep-ph/0001032.

[16] KORALW version 1.33 is used; S. Jadach et al., Comput. Phys. Commun. 94 (1996) 216; M. Jadach et al., Phys. Lett. B 372 (1996) 289.

[17] PHOJET version 1.05 is used; R. Engel, Z. Phys. C 66 (1995) 203; R. Engel et al., Phys. Rev. D 54 (1996) 4244.

[18] F.A. Berends et al., Nucl. Phys. B 253 (1985) 441.

[19] GEANT version 3.15 is used; R. Brun et al., Preprint CERN-DD/EE/84-1 (1984), revised 1987;

The GHEISHA program, H. Fesefeldt, RWTH Aachen Report PITHA 85/02 (1985), is used to simulate hadronic interactions. 